\title{
Viral evolution model with several time scales
}

\author{
A.A. Archibasov ${ }^{1}$

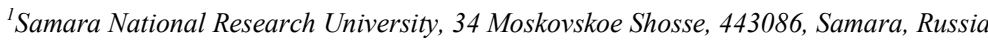

\begin{abstract}
In this paper a viral evolution model with specific immune response is considered. By introducing of dimensionless variables and parameters this model can be modified to the singularly perturbed system of partial integro-differential equations with two small parameters. The transition from the initial-boundary value problem of the initial system to the generating problem makes it possible to reduce the dimension of the system and, as a consequence, to reduce the computational volume. The theorem on the passage to the limit is also represented.
\end{abstract}

Keywords: viral dynamics; immune response; specific immunity; singular perturbations; initial-boundary value problem; degenerate system; passage to the limit

\section{Introduction}

The presence of several time scales in the models of evolution biology is more a rule than an exception. This is due to the fact that an extremely slow biological evolution process proceeds against the background of significantly faster interactions of different nature. To model such processes with several time scales, systems of differential equations with a small parameter for a part of the derivatives (the so-called singularly perturbed systems of differential equations) are usually used. Numerical analysis of such systems involves a large amount of computation due to the presence of variables that vary with significantly different velocities. Therefore, it becomes relevant to construct simplified (reduced) models of lower dimensionality, but with a high degree of accuracy reflecting the behavior of the original processes.

One of the reduction methods for singularly perturbed systems are the integral manifold method, developed in [1-3], and the passage to the limit to the solution of the degenerate system, used in present paper. In this case, the dimension of the systems under consideration is reduced. Below, this approach is used to reduce the dimension in the initial-boundary value problem for a system describing the dynamics of populations of healthy and infected cells and cytotoxic T-lymphocytes.

\section{Biological background}

A virus is a small infectious agent that is basically composed of a coat of protein, which covers a genetic code (DNA or RNA). A remarkable feature of viruses is inability to replicate themselves. Thus a virus particle attaches to a host cell and injects the genetic material into the cell. Further new virus particles released from the host cell and move around in the infected organism and infect new host cells. When a virus is replicated, mutations happen randomly. A mutant can be seen as a new strain of virus, where a viral strain is a genetic variant or subtype of a virus.

The immune system attacks a virus in order to stop it from growing or to kill it all together. The two main branches of the immune system are humoral and cell-mediated responses. The latter is composed of killer T-cells (also called cytotoxic Tlymphocytes, CTL). Specific immune cells can recognize the physical structure of a pathogen. When discovering the pathogen, the immune cells multiply rapidly in order to kill off the pathogen. Killer T-cells fight infected cells. CTL response is generally considered to be the most effective response of the immune system.

\section{Model}

Let us consider the model of viral dynamics with specific immune response [4]:

$$
\begin{aligned}
\frac{d u(t)}{d t} & =b-u(t) \int_{0}^{\infty} \beta(s) v(t, s) d s-c u(t), \\
\frac{\partial v(t, s)}{\partial t} & =\beta(s) u(t) v(t, s)-m v(t, s)+\mu \frac{\partial^{2} v(t, s)}{\partial s^{2}}-\xi v(t, s) z(t, s), \\
\frac{\partial z(t, s)}{\partial t} & =q v(t, s)+\gamma z(t, s)\left(1-\frac{z(t, s)}{p}\right)
\end{aligned}
$$

Each virus phenotype is described by a set of parameters and all possible values of these parameters form a phenotype space, which is assumed to be a one-dimensional and continuous: $s \in[0,+\infty)$ ( $s$ is a dimensionless quantity). Variables $v(t, s)$, cell $/ \mathrm{mm}^{3}$, and $z(t, s), c e l l / \mathrm{mm}^{3}$, are the population of infected cells of phenotype $s$ at a time $t$, day, and the population of CTL-cells, able to kill infected cells of phenotype $s$ at a time $t$, respectively. Uninfected target cells with concentration $u(t)$, cell $/ \mathrm{mm}^{3}$, are produced at constant rate $b, \operatorname{cell} /\left(\mathrm{mm}^{3} \cdot d a y\right)$, and have a natural death at a rate $c, 1 / d a y$. Uninfected cells become infected at a rate $\beta, \mathrm{mm}^{3} /($ virion $\cdot$ day $)$. The quantity $\operatorname{IF}(t)=\int_{0}^{+\infty} \beta(s) v(t, s) d s$ is called the infective force. Infected cells 
die naturally at a rate $m, 1 /$ day, and are eliminated by CTL response at a rate $\xi, \mathrm{mm}^{3} /($ virion-day). The activation term of CTL response is assumed to be proportional to $v(t, s)$ with a coefficient $q, 1 /$ day, since the number of infected cells has to be different from zero in order to activate the growth of $z(t, s)$. After activation of CTL response the activated cells will multiply by cloning (the so-called "clonal expansion"). To model this phenomenon, a logistic term is employed. Random mutations are described by the dispersion with a coefficient $\mu, 1 /$ day. Since $v(t, s)$ is a distribution, it is natural to assume that $v(t,+\infty)=0$.

The boundary condition at $s=0$ is the non-flux condition $\frac{\partial v}{\partial t}(t, 0)=0$ for convenience. Non-negative initial conditions at $t=0$ are $u(0)=u^{0}, v(0, s)=v^{0}(s)$ and $z(0, s)=z^{0}(s)$ (it is assumed that a host is already infected by a virus).

Without loss of generality, for simplicity, we assume that only $\beta$ depends on $s$ and that $m, \xi, q, \gamma$ are constant and have common values for all phenotypes.

Although the model is stated for $s \in[0,+\infty)$, the parameters $s$ is usually assumed to belong a finite interval $[0$,$] , and the$ boundary condition $v(t,+\infty)=0$ is replaced by the condition $\frac{\partial v}{\partial s}(t)=$,0 .

\section{The dimensionless system}

Let us introduce the following notations $t=T \bar{t}, s=S \bar{s}, u(T \bar{t})=\widetilde{U u}(\bar{t}), v(T \bar{t}, S \bar{s})=\widetilde{V} \bar{v}(\bar{t}, \bar{s}), z(T \bar{t}, S \bar{s})=\widetilde{Z z}(\bar{t}, \bar{s})$, and assume that $\mu T / S^{2}=1, \widetilde{U}=b / c, \widetilde{V}=(\gamma / q) \widetilde{Z}, \widetilde{Z}=p$. Then the initial-boundary value problem for model (1) takes the form

$$
\begin{gathered}
\varepsilon \frac{d \bar{u}(\bar{t})}{d \bar{t}}=1-\bar{u}(\bar{t}) \int \bar{\beta}(\bar{s}) \bar{v}(\bar{t}, \bar{s}) d \bar{s}-\bar{u}(\bar{t}), \\
\frac{\partial \bar{v}(\bar{t}, \bar{s})}{\partial \bar{s}}=\frac{\partial^{2} \bar{v}(\bar{t}, \bar{s})}{\partial \bar{s}^{2}}-\bar{m} \bar{v}+\bar{d} \bar{\beta}(\bar{s}) \bar{u}(\bar{t}) v(\bar{t}, \bar{s})-\bar{\xi} \bar{v}(\bar{t}, \bar{s}), \\
\varepsilon \theta \frac{\partial \bar{z}(\bar{t}, \bar{s})}{\partial \bar{t}}=\bar{v}(\bar{t}, \bar{s})+\bar{z}(\bar{t}, \bar{s})(1-\bar{z}(\bar{t}, \bar{s})), \\
\bar{u}(0)=\bar{u}^{0}, \quad \bar{v}(0, \bar{s})=\bar{v}^{0}(\bar{s}), \quad \frac{\partial \bar{v}}{\partial \bar{s}}(\bar{t}, 0)=0, \quad \frac{\partial \bar{v}}{\partial \bar{s}}\left(\bar{t}^{-}\right)=0, \quad \bar{z}(0, \bar{s})=\bar{z}^{0}(\bar{s}),
\end{gathered}
$$

where $\varepsilon=\frac{1}{c T}, \theta=\frac{c}{\gamma}, \bar{\beta}(\bar{s})=\frac{p S \gamma}{c q} \beta(S \bar{s}), \bar{m}=m T, \bar{d}=\frac{b q S}{p \gamma \mu}, \bar{\xi}=p T \xi, \bar{u}^{0}=\frac{c u^{0}}{b}, \bar{v}^{0}(\bar{s})=\frac{q}{p \gamma} v^{0}(S \bar{s}), \bar{z}^{0}(\bar{s})=\frac{z^{0}(S \bar{s})}{p}$, $-\bar{S}$.

The parameter $T$ must be taken so that the inequality $\varepsilon<<1$ holds. For example, $T=1 / \mu$, then $S=\sqrt{\mu T}=1$. The parameter $\mu$ is proportional to the mutation probability. For HIV $\mu$ does not exceed $10^{-7}-10^{-9} \quad 1 /$ day, and HIV is known as one of the most rapidly mutating RNA-viruses, so that $\varepsilon$ is substantially smaller for more slowly mutating RNA-viruses (and so much the more DNA-viruses). As $c<<\gamma$, then $\theta<<1$. Thereby system (2) is a singularly perturbed system with two small parameters and as result has three time scales. It should be noted that a system with several time scales was considered in the original work [5]. Further to simplify the notation, we omit the bar.

\section{Reduction of dimension}

Setting $\theta=0$ in (2), we obtain the so-called first-order degenerate system

$$
\begin{aligned}
\varepsilon \frac{d u}{d t} & =1-u \int_{0} \beta v d s-u, \\
\frac{\partial v}{\partial t} & =\frac{\partial^{2} v}{\partial s^{2}}-m v+d \beta u v-\xi v z, \\
0 & =v+z(1-z) .
\end{aligned}
$$

The third equation is algebraic and has two roots $z_{1,2}=(1 \pm \sqrt{1+4 v}) / 2$. For the first-order associated system

$$
\frac{\partial \hat{z}}{\partial \tau}=\hat{z}(1-\hat{z})+v
$$

where $v$ enters as a parameter, only one of the roots, namely $z=\varphi(v)=(1+\sqrt{1+4 v}) / 2$, is the asymptotically stable (in the sense of Lyapunov) stationary point, because $\left.\frac{\partial}{\partial \hat{z}}(\hat{z}(1-\hat{z}))\right|_{\hat{z}=\varphi(v)}=-\sqrt{1+4 v}<0$.

At the initial value of the parameter $v$, i.e., at $v=v^{0}(s)$, the system (5) with the initial condition $\hat{z}(0, s)=z^{0}(s)$ has a unique solution $\hat{z}(\tau, s)$ for $\tau \geq 0$, and besides $\lim _{\tau \rightarrow+\infty} \hat{z}(\tau, s)=\varphi\left(v^{0}(s)\right) \quad \forall s \in[0$,$] (see Appendix). Thereby the initial point z^{0}(s)$ of the 
first-order associated system (5) belongs to the domain of attraction of the stable stationary point $\varphi\left(v^{0}(s)\right)$. Thus, for sufficiently small $\theta$, problem (2), (3) has a unique solution and, for some $t_{1}$, the following limiting equalities hold [6]:

$$
\begin{aligned}
\lim _{\theta \rightarrow+0} u(t, \varepsilon, \theta) & =u_{0}(t, \varepsilon) \quad \text { for } \quad 0 \leq t \leq t_{1}, \\
\lim _{\theta \rightarrow+0} v(t, s, \varepsilon, \theta) & =v_{0}(t, s, \varepsilon) \quad \text { for } \quad 0 \leq t \leq t_{1}, 0 \leq s \leq, \\
\lim _{\theta \rightarrow+0} z(t, s, \varepsilon, \theta) & =\varphi\left(v_{0}(t, s, \varepsilon)\right) \quad \text { for } \quad 0<t \leq t_{1}, 0 \leq s \leq,
\end{aligned}
$$

where $u(t, \varepsilon, \theta), v(t, s, \varepsilon, \theta), z(t, s, \varepsilon, \theta)$ are the solutions of the system $(2)$ and $u_{0}(t, \varepsilon), v_{0}(t, s, \varepsilon)$ are the solutions of the system (4). Note that the third limiting equality holds for $t \neq 0$, as the solution $z=\varphi(v)$ of reduced system (4), generally speaking, does not satisfy initial condition for this variable in (3). The boundary layer phenomenon occurs. Equation (5) is also called the boundary layer equation. Naturally, there is no boundary layer if the initial point falls on the slow surface [7-9]. The system (4) has a dimension one less in comparison with (2).

Let $\varepsilon=0$ in (4). Then we obtain the second-order degenerate system

$$
\begin{aligned}
0 & =1-u \int_{0} \beta v d s-u, \\
\frac{\partial v}{\partial t} & =\frac{\partial^{2} v}{\partial s^{2}}-m v+d \beta u v-\xi v z, \\
0 & =v+z(1-z)
\end{aligned}
$$

first equation in which is algebraic with respect to $u$ and has a root $u=\psi(v)=1 /\left(1+\int_{0} \beta v d s\right)$. This root is the asymptotically stable (in the sense of Lyapunov) stationary point of the second-order associated system

$$
\frac{d \hat{u}}{d \tau}=-\left(1+\int_{0} \beta v d s\right) \hat{u}+1
$$

The latter equation with the initial condition $\hat{u}(0)=u^{0}$ at the initial value of the parameter $v=v^{0}(s)$ has a unique solution $\hat{u}(\tau)=\left(u^{0}-1 / f\right) e^{-f \tau}+1 / f, f=\psi\left(v^{0}(s)\right)=1+\int_{0} \beta(s) \nu^{0}(s) d s$, for all $\tau \geq 0$ and $\lim _{\tau \rightarrow+\infty} \hat{u}(\tau)=1 / f$. Thus, the initial point $u^{0}$ of the second-order associated system (8) belongs to the domain of attraction of the stable stationary point $\psi\left(v^{0}(s)\right)$. Consequently, for some $t_{2}$

$$
\begin{aligned}
\lim _{\varepsilon \rightarrow+0} u_{0}(t, \varepsilon) & =\psi\left(v_{00}(t, s)\right) \quad \text { for } \quad 0<t \leq t_{2}, \\
\lim _{\varepsilon \rightarrow+0} v_{0}(t, s, \varepsilon) & =v_{00}(t, s) \quad \text { for } \quad 0 \leq t \leq t_{2}, 0 \leq s \leq, \\
\lim _{\varepsilon \rightarrow+0} z_{0}(t, s, \varepsilon) & =\varphi\left(v_{00}(t, s)\right) \quad \text { for } \quad 0<t \leq t_{2}, 0 \leq s \leq,
\end{aligned}
$$

where $v_{00}(t, s)$ is the solution of the second equation in (7) with boundary and initial conditions for variable $v$ in (3). The passage to the limit for $u_{0}$ is not carried out at point $t=0$. As a result, a system of three integro-differential equations reduces to one integro-differential equation. The existence and uniqueness of the solution of the initial value problem for integroparabolic equation in (7) can be justified with the use of the approach outlined in the monograph [10].

\section{Admissibility of the passage to the limit}

In work [6] the theorem, that connects the solutions of the singularly perturbed system of partial integro-differential equations with one small parameter, is proved. Generalize this theorem to the case of two small parameters.

Consider the singularly perturbed system of partial integro-differential equations

$$
\begin{aligned}
\varepsilon \frac{d u}{d t} & =f\left(u, \int_{0} g(s, v) d s\right), \\
\varepsilon \theta \frac{\partial z}{\partial t} & =h(z, v), \\
\frac{\partial v}{\partial t} & =\frac{\partial^{2} v}{\partial s^{2}}+q(s, u, z, v)
\end{aligned}
$$

with the initial and boundary conditions

$$
u(0)=u^{0}, \quad z(0, s)=z^{0}(s), \quad v(0, s)=v^{0}(s), \quad \frac{\partial v}{\partial s}(t, 0)=0, \quad \frac{\partial v}{\partial s}(t,)=0,
$$

where $u, z, v \in R, t \in R, 0<\varepsilon, \theta<<1$ are the small positive parameters.

We assume that system (10) satisfies the following conditions.

I. The functions $f(u, x), g(s, v), h(z, v)$, and $q(s, u, z, v)$, together with their partial derivatives with respect to all variables, are uniformly continuous and bounded in the respective domains $\Omega_{1}=\{|u| \leq a,|x| \leq b\}, \quad \Omega_{2}=\{0 \leq s \leq,|v| \leq c\}$, $\Omega_{3}=\{|z| \leq d,|v| \leq c\}, \Omega_{4}=\{0 \leq s \leq,|u| \leq a,|z| \leq d,|v| \leq c\}$. 
II. The equation $h(z, v)=0$ has an isolated root $z=\varphi(v)$ in the domain $\{|v| \leq c\}$ and in this domain function $z=\varphi(v)$ is continuously differentiable.

III. The inequality $h_{z}(\varphi(v), v) \leq-\alpha<0$ holds for $|v| \leq c$. This condition implies that the stationary point $\hat{z}=\varphi(v)$ of the firstorder associated system

$$
\frac{\partial \hat{z}}{\partial \tau}=h(\hat{z}, v)
$$

which contains $v$ as a parameter, is Lyapunov asymptotically stable as $\tau \rightarrow+\infty$ uniformly with respect to $v$, $|v| \leq c$. If assumption III is satisfied, then we say for brevity that the zero of the function $\varphi(v)$ is stable.

IV. There exist a solution $\hat{z}(\tau)$ of the problem

$$
\frac{\partial \hat{z}}{\partial \tau}=h\left(\hat{z}, v^{0}(s)\right), \quad \hat{z}(0, s)=z^{0}(s)
$$

for $\tau \geq 0,0 \leq s \leq$. Further, this solution tends to the stationary point $\varphi\left(v^{0}(s)\right)$ as $\tau \rightarrow+\infty$, i.e. $z^{0}(s)$ belongs to the domain of attraction of the stable stationary point $\varphi\left(v^{0}(s)\right)$.

$\mathrm{V}$. The equation $f(u, x)=0$ has an isolated root $u=\psi(x)$ in the domain $\{|x| \leq b\}$ and in this domain function $u=\psi(x)$ is continuously differentiable.

VI. The inequality $f_{u}(\psi(x), x) \leq-\beta<0\left(x=\int_{0} g(s, \varphi(v)) d s\right)$ holds for $|v| \leq c$, i.e. the stationary point $\hat{u}=\psi(x)$ of the secondorder associated system

$$
\frac{d \hat{u}}{d \tau}=f\left(\hat{u}, \int_{0} g(s, \varphi(v)) d s\right)
$$

which contains $v$ as a parameter, is Lyapunov asymptotically stable as $\tau \rightarrow+\infty$ uniformly with respect to $v$, $|v| \leq c$. If assumption VI is satisfied, then we say for brevity that the zero of the function $\psi(x)$ is stable.

VII. There exist a solution $\hat{u}(\tau)$ of the problem

$$
\frac{d \hat{u}}{d \tau}=f\left(\hat{u}, \int_{0} g\left(s, \varphi\left(v^{0}(s)\right)\right) d s\right), \quad \hat{u}(0)=u^{0},
$$

for $\tau \geq 0$. Further, this solution tends to the stationary point $\psi\left(\int_{0} g\left(s,\left(\varphi\left(v^{0}(s)\right)\right)\right) d s\right)$ as $\tau \rightarrow+\infty$, i.e. $u^{0}$ belongs to the domain of attraction of the stable stationary point.

VIII. The truncated system

$$
\begin{aligned}
\frac{\partial v}{\partial t} & =\frac{\partial^{2} v}{\partial s^{2}}+q(s, \psi(x), \varphi(v), v), \\
u & =\psi(x), \\
z & =\varphi(v), \\
x & =\int_{0} g(s, \varphi(v)) d s, \\
v(0, s) & =v^{0}(s), \quad \frac{\partial v}{\partial s}(t, 0)=0, \quad \frac{\partial v}{\partial s}(t,)=0,
\end{aligned}
$$

has a unique solution $\bar{v}(t, s), \bar{u}(t)=\psi\left(\int_{0} g(s, \varphi(\bar{v}(t, s))) d s\right), \bar{z}(t, s)=\varphi(\bar{v}(t, s))$.

Theorem. If conditions I-VII are satisfied, then, for sufficiently small $\varepsilon, \theta$, problem (10), (11) has a unique solution $u(t, \varepsilon, \theta), z(t, s, \varepsilon, \theta), v(t, s, \varepsilon, \theta)$, which is related to the solution $\bar{u}(t), \bar{z}(t, s), \bar{v}(t, s)$ of the truncated problem (16), (17) by the limit formulas

$$
\begin{aligned}
\lim _{\substack{\varepsilon \rightarrow+0 \\
\theta \rightarrow+0}} u(t, \varepsilon, \theta) & =\bar{u}(t)=\psi\left(\int_{0} g(s, \varphi(\bar{v}(t, s))) d s\right), \quad 0<t \leq T, \\
\lim _{\substack{\varepsilon \rightarrow+0 \\
\theta \rightarrow+0}} z(t, s, \varepsilon, \theta) & =\bar{z}(t, s)=\varphi(\bar{v}(t, s)), \quad 0<t \leq T, 0 \leq s \leq, \\
\lim _{\substack{\varepsilon \rightarrow+0 \\
\theta \rightarrow+0}} v(t, s, \varepsilon, \theta) & =\bar{v}(t, s), \quad 0<t \leq T, 0 \leq s \leq,
\end{aligned}
$$

Here $T$ is an arbitrary number such that $u=\psi\left(\int_{0} g(s, \varphi(\bar{v}(t, s))) d s\right), z=\varphi(\bar{v}(t, s))$ are the isolated stable roots of the equations $f\left(u, \int_{0} g(s, \varphi(\bar{v}(t, s))) d s\right)=0, h(\varphi(\bar{v}(t, s)), \bar{v}(t, s))=0$ for $0 \leq t \leq T$ accordingly.

The proof of this theorem is the same as one in [6]. 


\section{Conclusion}

In this paper the procedure that the original system of three integro-differential equations reduces to a single integrodifferential equation is given for a viral evolution model with specific immune response. The theorem on the passage to the limit is also formulated. The limiting equalities for fast variables whose physical meaning is the concentration of populations of healthy cells and killer T-cells are valid only for some segment $[\delta, T], \delta>0$, separated from zero. To construct an approximate solution in a neighborhood of the point $t=0$ Tikhonov-Vasil'eva boundary function method [11] can be applied. In the paper [12] a model of viral evolution without immune response (but this model is described by a system of the same type which this work deals with) was considered. By the method mentioned above the solutions in powers of small parameters were found.

It should be noted that the mathematical models of evolution biology are usually formulated as integro-differential equations and PDE. Thus the same concept and the same techniques can be used to a model of evolution based on any other model virus dynamics.

\section{Acknowledgements}

The study was supported by the Russian Foundation for Basic Research and Samara region (grant 16-41-630529-p) and the Ministry of Education and Science of the Russian Federation as part of a program to increase the competitiveness of SSAU in the period 2013-2020.

\section{Appendix}

Let us solve the initial value problem

$$
\frac{\partial \hat{z}}{\partial \tau}=\hat{z}(1-\hat{z})+v^{0}(s), \quad \hat{z}(0, s)=z^{0}(s)
$$

where $\hat{z}(\tau, s)$ is unknown function, functions $v^{0}(s), z^{0}(s)$ are given. This equation is the Riccati equation. Performing successively in the equation of change of variables $\hat{z}=\hat{z}_{1}+\sqrt{1+v^{0}(s)} / 2, y=1 / \hat{z}_{1}$, we first bring it to the Bernoulli equation, and then to a linear nonhomogeneous equation of the first order

$$
\frac{\partial y}{\partial \tau}=y \sqrt{1+4 v^{0}(s)}+1
$$

with the initial condition $y(0, s)=1 /\left(z^{0}(s)-\varphi\left(v^{0}(s)\right)\right)$, where $\varphi(v)=(1+\sqrt{1+4 v}) / 2$. Solving this linear equation by the variation method, we find that $y(\tau, s)=\frac{1}{2 \varphi^{0}-1}\left(e^{\left(2 \varphi^{0}-1\right) \tau}-1\right)+\frac{1}{z^{0}-\varphi^{0}}, \varphi^{0}=\varphi\left(v^{0}(s)\right), z^{0}=z^{0}(s)$, then $\hat{z}(\tau, s)=\varphi^{0}+\frac{1}{y(\tau, s)} \rightarrow \varphi^{0}$ as $\tau \rightarrow+\infty$

\section{References}

[1] Shchepakina E, Sobolev V, Mortell MP. Reduction methods for chemical systems. Singular Perturbations. Introduction to System Order Reduction Methods with Applications. Lecture Notes in Mathematics 2014; 2114: 111-117.

[2] Shchepakina E, Korotkova O. Condition for canard explosion in a semiconductor optical amplifier. Journal of the Optical Society of America B: Optical Physics 2011; 28(8): 1988-1993.

[3] Shchepakina E, Korotkova O. Canard explosion in chemical and optical systems. Discrete and Continuous Dynamical Systems. Series B 2013; 18(2): 495-512.

[4] Laarhoven N, Korobeinikov A. Within-Host Viral Evolution Model with Cross-Immunity. Extended Abstracts Spring 2015; 119-124. DOI: 10.1007/9783-319-22129-8 21.

[5] Tikhonov AN. Systems of differential equations with small parameters multiplying the derivatives. Mat. Sb. 1952; 31: 575-586.

[6] Archibasov AA, Korobeinikov A, Sobolev VA. Pasage to the limit in a singularly perturbed partial integro-differential system. Differential Equations 2016; 52(9): 115-1122. DOI: $10.1134 / \mathrm{S} 0012266116090020$.

[7] Shchepakina E, Sobolev V, Mortell MP. Slow integral manifolds. Singular Perturbations. Introduction to System Order Reduction Methods with Applications. Lecture Notes in Mathematics 2014; 2114: $25-42$.

[8] Shchepakina E, Sobolev V. Invariant surfaces of variable stability. Journal of Physics: Conference Series 2016; 727(1).

[9] Shchepakina E. Stable/unstable slow integral manifolds in critical cases. Journal of Physics: Conference Series 2017; 811.

[10] Henry D. Geometric theory of semilinear parabolic equations. Berlin: Springer-Verlag, 1981; 358 p.

[11] Vasil'eva AB, Butuzov VF, Kalachev LV. The boundary function method for singular perturbation problems. Philadelphia: SIAM, 1995; 236 p.

[12] Archibasov AA, Korobeinikov A, Sobolev VA. Asimptotic expansions of solutins in a singularly perturbed model of virus evolution. Computational Mathematics and Mathematical Physics 2015; 55(2): 240-250. DOI:10.1134/S0965542515020037. 